\title{
SEQUENTIAL COMPUTATION OF THE COMPLETE EIGENSYSTEM FOR THE STUDY ZONE IN SMALL SIGNAL STABLITY ANALYSIS OF LARGE POWER SYSTEMS
}

\author{
Adam Semlyen \\ Lei Wang \\ Department of Electrical Engineering \\ University of Toronto \\ Toronto, Ontario, Canada
}

\begin{abstract}
This paper presents an efficient method for the calculation of the complete set of eigenvalues and eigenvectors associated with the state variables in a specified study zone of a large power system, linearized for the purpose of small signal stability studies. The study zone is assumed to be within a wider area, the nucleus, and the latter surrounded by the external system.

The computation is performed in two stages. Stage 1 starts with a set of first guesses for the eigenvalues, and reduces the full system model to the size of the nucleus for which a QR algorithm gives all eigenvalues and eigenvectors, to be used as starting values in Stage 2. This uses the augmented system state equations to refine the eigenpairs, by means of the inverse power method, Newton's method and deflation techniques. The computations are very efficient due to the reduced order of the eigenproblem in Stage 1 and the sparsity of the augmented system state equations in Stage 2.

The computer implementation STEPS (Sequential Two stage Eigenanalysis for Power Systems) is based on the described procedures. Illustrative results are given for a 117 bus test system with 30 machines.
\end{abstract}

\section{INTRODUCTION}

The evaluation of the small signal (steady state) stability of power systems requires the calculation of the eigenvalues, and sometimes also of the eigenvectors, of a very large unsymmetrical and nonsparse matrix. The well known $Q R$ algorithm is robust and converges fast ${ }^{1}$ but can not be used for the state matrices of large power systems (of order larger than, say, $500 \times 500$ ). Therefore, significant effort has been expended to solve the problem by different means. The usual approach would be to calculate only some eigenpairs (eigenvalues and related eigenvectors), and this is normally achieved by means of the inverse power method. It is particularly simple to calculate the mechanical modes only, because in a physical sense these represent the basic links between machines and all other modes are connected indirectly to each other only through these modes. Such an approach has been reported in Ref.[2] and [3]. Ref. [4] gives an overview of the state of the art of sta-

This paper was sponsored by the IEEE Power Engineering Society for presentation at the IEEE Power Industry Computer Application Conference, Montreal, Canada, May 18-21, 1987. Manuscript was published in the 1987 PICA Conference Record. bility calculations. Ref.[5] presents a method for obtaining a selected set of eigenpairs. The method is, however of restricted applicability because it does not use sparsity techniques. This disadvantage is overcome in the method of Ref.[6], by augmenting the system state equations, or equivalently, by retaining the load buses of the system. The method does not provide appropriate starting procedures to ensure that all desired eigenpairs can be found. The recent reference [7] describes an adaptation of the basic technique of Ref.[3] to very large systems by using a very efficient sparsity based solution method. It is aimed at the computation of slow interarea oscillatory modes.

The method presented in this paper has two stages of computations, with a set of first guesses for the eigenpairs. The system is first divided into two areas: the nucleus which includes the study zone and the external system, and then linearized to obtain the augmented system state equations. Stage 1 uses a first rough guess of eigenvalues in the external system and in some places in the nucleus, so that the order of the problem is significantly reduced. The $Q R$ algorithm, applied to the reduced order problem, yields a set of eigenpairs, to be refined in Stage 2. There the augmented system state equations are used, to obtain the accurate eigenpairs related to the state variables of the study zone. The inverse power method, Newton's method, and deflation techniques are used for fast and reliable convergence. Since the augmented system state equations are very sparse, the algorithm for Stage 2 is very efficient and saves much storage.

We call STEPS (Sequential Two-stage Eigenanalysis for Power Systems) the computer implementation of the pro cedures described in this paper.

Finally, a numerical example is given for a 117 bus, 30 machine system, to illustrate the method.

\section{DESCRIPTION OF THE METHOD}

\section{Problem Definition}

The purpose of this work is to calculate the complete eigensystem for the study zone of a large power system. We define the study zone as a small part of the system, consisting of a few machines in which we are interested. By complete eigensystem of the study zone we mean the set of all eigenpairs which are dominant in at least one of the state variables of the study zone. Dominance is measured here by the participation factor of the respective eigenvalue in the state variable being considered; see Appendix 1 and Ref.[5]. This implies that, for each state variable, eigenvalues with a participation factor not less than a specified threshold value $\alpha$ (say, 0.05) will be considered as dominant.

0885-8950/88/0500-0715\$01.00@1988 IEEE 
Fundamental to the method presented here is the idea that modes related to distant machines do not have to be represented accurately for a first evaluation of the eigensystem of the study zone. The structure of a power system leads to a grouping of the modes into two categories: mechanical modes (related to angles and angular velocities) and non-mechanical modes (related to all other state variables). The latter are essentially local, as they are linked to each other only through the mechanical state variables. These, however, are directly connected through the synchronizing powers of the transmission links. Therefore, it would appear that, for the mechanical modes, the complete system has to be included in the eigenvalue evaluation. However, even synchronizing links via transmission lines transmit effects only to some neighbourhood of a centre of disturbance. Considering this fact, it is not necessary to represent the whole system accurately for a preliminary evaluation of the eigensystem related to the study zone. It is sufficient to interpose between the study zone and the external system a buffer area so that the complete system can be divided into a nucleus, which includes the study zone and a buffer area, and the external system; see Fig. 1.

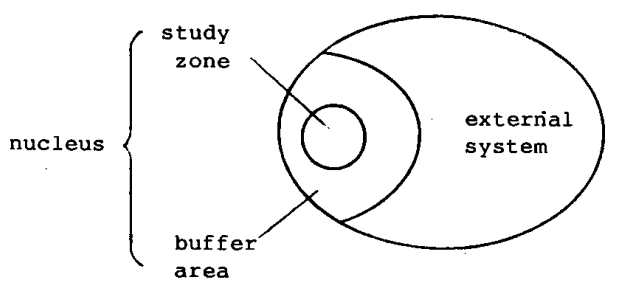

Fig. 1 Representation of study zone, nucleus and external system

It is important to note that the components in the external system can be represented with any degree of sophistication (or simplicity), as required by the type of study. For example, if interarea oscillatory modes are of interest, the detailed modelling of such components may be quite significant, as pointed out in Ref.[7].

\section{Overview of the Method}

With the ideas of nucleus and external system, we solve the problem in two stages, as follows.

Stage 1- The complete system is first linearized around an operating point, to obtain the augmented system state equations which include both differential and algebraic equations. This system of equations is very sparse and is made completely algebraic by replacing $d / d t$ by $\lambda$ for the eigenproblem. Next, we partition the equations, as discussed in more detail in the section below, and replace in those corresponding to the external system and in some places in the nucleus a first guess for $\lambda$, while keeping $\lambda$ as a variable elsewhere. The part not containing $\lambda$ is then eliminated and further simplifications are made for different kinds of eigenvalues, so that a very low order eigenproblem is obtained in standard form. This is solved by a QR algorithm for both eigenvalues and eigenvectors. This procedure is repeated for several first guess values for $\lambda$ to produce sufficient starting values for the eigenpairs to be used in Stage 2.
Stage 2- This serves to refine an eigenpair obtained at Stage 1. Clearly, the complete set of equations (that is, the augmented system state equations) has to be used for this purpose. Two main procedures of refinement have been used: the inverse power method and Newton's method. Since some of the eigenvalues in a large power system may be very close, in order to prevent convergence to the same value, deflation has been successfully used.

When an eigenpair has been calculated, the participation factors related to the state variables of the study zone are evaluated in order to see whether the calculated mode is dominant and to assess whether all dominant modes pertaining to the study zone have been found.

\section{Sparse Problem Formulation}

We assume that the system to be modelled has $N$ buses and $M$ machines. For simplicity, the loads are represented as constant impedances and no other dynamic components are considered in the study.

For machine no.i, we write the linearized state equation

$$
p x_{i}=J_{A_{i}} x_{i}+J_{B_{i}}\left[\begin{array}{c}
V_{R_{i}} \\
V_{I_{i}}
\end{array}\right]
$$

and the output equation

$$
\left[\begin{array}{l}
I_{R_{i}} \\
I_{L_{i}}
\end{array}\right]=-J_{C_{i}} x_{i}+J_{D_{i}}\left[\begin{array}{c}
V_{R_{i}} \\
V_{I_{i}}
\end{array}\right]
$$

where

$x_{i}$ represents the state vector,

$V_{R_{i}}$ and $V_{L_{i}}$ represent the real and imaginary part of the machine bus roltages,

$I_{R_{i}}$ and $I_{I_{i}}$ represent the real and imaginary part of currents injected into the system,

$J_{A_{i}}, J_{B_{i}}, J_{C_{i}}$ and $J_{D_{i}}$ represent appropriate Jacobian matrices,

all for machine no.i. The size of eqn.(1) depends on the model used for the machine and can vary within a wide range.

For the network, including all buses, we have

$$
Y V=I
$$

where

$$
\begin{gathered}
V=\left[\begin{array}{lllll}
V_{R_{1}} & V_{I_{1}} & \cdots & V_{R_{N}} & V_{I_{N}}
\end{array}\right]^{T} \\
I=\left[\begin{array}{lllll}
I_{R_{1}} & I_{I_{1}} & \cdots & I_{R_{N}} & I_{I_{N}}
\end{array}\right]^{T}
\end{gathered}
$$

and $Y$ represents the system admittance matrix in terms of conductances and susceptances.

We note that in all buses, except generator nodes, the current injections are zero. Therefore, eqns.(1) - (3) are enough for forming the complete set of system equations.

Combining eqn.(1) through (3), we obtain for the complete system

$$
\left[\begin{array}{c}
p x \\
0
\end{array}\right]=\left[\begin{array}{ll}
J_{A} & J_{B} \\
J_{C} & J_{D}
\end{array}\right]\left[\begin{array}{l}
x \\
V
\end{array}\right]
$$

where $x=\left[x_{1}^{T} \cdots x_{M}^{T}\right]^{T}$ is the complete state vector. The coefficient matrix of eqn.(5) has the following structure: 
$J_{A}$ is block-diagonal, with $J_{A_{i}}$ on the diagonal,

$J_{B}$ consists of the blocks $J_{B_{i}}$ in the columns related to the, machine buses and is empty elsewhere,

$J_{C}$ consists of the blocks $J_{C_{i}}$ and is structurally symmetrical to $J_{B}$,

$J_{D}$ is the sparse matrix $Y$ of eqn.(3), with the $2 \times 2$ blocks $J_{D_{i}}$ subtracted on the diagonal.

We note that the coefficient matrix of eqn.(5) is very sparse, even though it has an extremely big size $(=$

$2 \times N+\sum d_{i}$, where $d_{i}$ is the model order of machine no.i). We

call eqn.(5) the augmented system state equations.

From eqn.(5) we could, in principle, obtain the system state matrix

$$
A=J_{A}-J_{B} J_{D}^{-1} J_{C}
$$

for subsequent computations. However, the matrix $A$ is not sparse and therefore is practically worthless for the purpose of this work. Instead, we will always use the augmented system state equations in the cases where the complete system equations are needed.

For the purpose of eigenanalysis, we will replace in the forthcoming computations, $p=d / d t$ by $\lambda$.

\section{Stage 1: Obtaining Starting Values for Eigenpairs}

In order to reduce the dynamic order of eqn.(5) we first replace $\lambda$ by a first guess $\lambda_{0}$ for all state variables related to machines in the external system. Arranging the order of all machines so that the machines in the nucleus come first, we obtain from eqn.(5) the partitioned equation

$$
\left[\begin{array}{c}
\lambda x^{\prime} \\
\hline 0 \\
0
\end{array}\right]=\left[\begin{array}{c|cc}
J^{\prime} & 0 & J^{\prime}{ }_{B} \\
\hdashline 0 & J^{\prime}{ }_{A}-\lambda_{0} I & J^{\prime}{ }_{B} \\
J_{C}^{\prime} & J^{\prime}{ }_{C} & J_{D}
\end{array}\right]\left[\begin{array}{l}
x^{\prime} \\
V
\end{array}\right]
$$

where, in the external system we have replaced $\lambda$ by $\lambda_{0}$. To eliminate the second part of eqn.(7), we have to solve sequentially the sparse equations of the form:

$$
\left[\begin{array}{cc}
J^{\prime}{ }_{A}-\lambda_{0} I & J^{\prime \prime}{ }_{B} \\
J^{\prime}{ }_{C} & J_{D}
\end{array}\right]\left[\begin{array}{l}
z \\
v
\end{array}\right]=\left[\begin{array}{l}
w \\
u
\end{array}\right]
$$

A special algorithm for solving this equation is presented in Appendix 2.

We note that the above elimination reduces the dynamic size of the system to that of the nucleus, for which the full order representation of the eigenproblem is feasible.

Next, we replace $\lambda$ by the same $\lambda_{0}$ even for some state variables inside the nucleus, to obtain a further reduction. This is done differently for different modes and is explained in the following.

We group the modes in three categories:

- mechanical, with frequency of the order of $2 \pi \mathrm{rad} / \mathrm{s}$,

-slow, i.e. $|\lambda|<\approx 2 \pi$,

-fast, i.e. $|\lambda|>\approx 2 \pi$.

For mechanical modes, we consider only the mechanical equations and without frictional losses. Then the angular velocity is no longer a state variable and the eigenvalues appear in the form $\lambda^{2}$. Let $\lambda^{\prime}=\lambda^{2}$. Then we have an eigenproblem of order $m$ in $\lambda^{\prime}$, where $m$ is the number of machines in the nucleus. For all other state variables we use the first guess $\lambda_{0}=j 2 \pi$.

For slow eigenvalues the inertia of the machines is not too significant and, therefore, we keep the variable $\lambda$ only with the state variables of the study zone and with the $\delta$ 's of the machines in the nucleus. Elsewhere we use $\lambda_{0}$, with a small value. Thus, the size of the eigenproblem is somewhat greater than $m$, but still of order $m$, since in large power systems the nucleus is much bigger than the study zone.

For fast eigenvalues the inertia is important. Therefore, we keep the variable $\lambda$ with the state variables of the study zone and with the $\omega$ 's of the machines in the nucleus. In all other places we use a large value as the first guess. The size of the problem is again of order $m$, as before.

For the final eigenproblem of order $m$, we use the $Q R$ algorithm to calculate the complete set of eigenpairs, of which a selected set will serve as starting values in Stage 2 .

The procedures discussed here will normally lead to improved starting values for Stage 2 since it can be considered as one step of a convergent iterative process, examined in our discussion to Ref.[7].

\section{Stage 2: Refining the Eigenpairs}

There are several methods, presented in the literature ${ }^{8,9}$, for refining eigenpairs. We shall describe below the methods we have used in STEPS, with the extensions needed due to the special structure of our problem.

\section{The Inverse Power Method}

In this procedure an eigenvector is updated by solving the equation

$$
\left(A-\lambda_{k} I\right) x_{k+1}=x_{k}
$$

for $x_{k+1}$. In eqn.(9) $\lambda_{k}$ is the estimate of the eigenvalue at step $k$. It can be kept the same over several steps or it can be updated using the Rayleigh quotient. We normalize $x_{k+1}$ to have unity as its largest element.

In our case, the iteration algorithm in eqn.(9) is in the augmented form below, obtained from eqn.(5),

$$
\left[\begin{array}{cc}
J_{A}-\lambda_{k} I & J_{B} \\
J_{C} & J_{D}
\end{array}\right]\left[\begin{array}{c}
x_{k+1} \\
v
\end{array}\right]=\left[\begin{array}{c}
x_{k} \\
0
\end{array}\right]
$$

where $v$ is not of direct interest. This equation has the same structure as eqn.(8) and is solved in a similar way.

The inverse power method will normally converge with any starting vector. If the shift $\lambda_{k}$ is kept constant, the convergence is only linear. If it is updated at each step using the Rayleigh quotient, the convergence becomes quadratic, but the coefficient matrix becomes ill-conditioned as the solution is approached. Therefore, this method will be used to start up the refinement process. We also note that, with an appropriate shift to start with, it is possible that changing the shift in subsequent steps will move it in such a way that the method will eventually converge to an undesired value.

\section{Newton's Method}

The eigenanalysis problem consists in the solution of the 
nonlinear equation

$$
f(\lambda, x)=A x-\lambda x=0
$$

with some normalization constraint on $x$ to guarantee a unique solution for an eigenpair. Accordingly, we will consider the starting vector $x$ being normalized to have unity as its largest element. During the iteration process this element will remain unchanged, while others will change and possibly exceed unity.

Newton's method uses the linearized form of eqn.(11):

$$
f\left(\lambda_{k}, x_{k}\right)+\left.\frac{\partial f}{\partial \lambda}\right|_{k} \Delta \lambda+\left.\frac{\partial f}{\partial x}\right|_{k} \Delta x=0
$$

or

$$
-x_{k} \Delta \lambda+\left(A-\lambda_{k} I\right) \Delta x=-f_{k}
$$

Eqn.(12b) can be written in the form

$$
B \Delta x^{\prime}=-f_{k}
$$

where $\Delta x^{\prime}$ is a vector containing all elements of $\Delta x$, except the one corresponding to the unity element of $x$, and having instead $\Delta \lambda$ at that location. Correspondingly, the Jacobian matrix $B$ contains essentially the matrix $A-\lambda_{k} I$ of eqn.(12b), except for the column corresponding to $\Delta \lambda$ which is replaced by $-x_{k}$.

To preserve sparsity, we again write eqn.(11) in the augmented form

$$
f_{a}(\lambda, x, v)=\left[\begin{array}{cc}
J_{A}-\lambda I & J_{B} \\
J_{C} & J_{D}
\end{array}\right]\left[\begin{array}{l}
x \\
v
\end{array}\right]=0
$$

from which we obtain the linearized equation

$$
\left[\begin{array}{cc}
J_{A}-\lambda_{k} I & J_{B} \\
J_{C} & J_{D}
\end{array}\right]\left[\begin{array}{c}
\Delta x \\
\Delta v
\end{array}\right]+\left[\begin{array}{c}
-x_{k} \\
0
\end{array}\right] \Delta \lambda=-f_{a_{k}}
$$

We note that the coefficient matrix of eqn.(15) becomes illconditioned near the solution. However, if we replace one of its columns by the coefficient vector of $\Delta \lambda$, as in eqn.(13), the sparsity of the system would be impaired. Therefore, we replace it by a unit vector $e$ (with unity at the location corresponding to the diagonal of the matrix) and denote the resulting matrix by $J_{k}$. Consequently, we add a correction matrix corresponding to the difference $g_{k}$ between the coefficient vector of $\Delta \lambda$ and the vector $e$. Then eqn.(15) becomes

$$
\left[J_{k}+g_{k} e^{T}\right]\left[\begin{array}{c}
\Delta x \\
\Delta v
\end{array}\right]=-f_{a_{k}}
$$

where $\Delta x^{\prime}$ is identical to that in eqn.(13). In eqn.(16) the matrix $g_{k} e^{T}$ is of unity rank. Therefore, only the sparse matrix $J_{k}$ (which has the same sparse structure as the coefficient matrix in eqn.(15)) has to be factorized and the complete solution is obtained, using the Matrix Modification Lemma $^{11}$, as

$$
\left[\begin{array}{c}
\Delta x \\
\Delta v
\end{array}\right]=-J_{k}^{-1} f_{a_{k}}+\frac{\left(J_{k}^{-1} g_{k}\right)\left(e^{T} J_{k}^{-1} f_{a_{k}}\right)}{1+e^{T} J_{k}^{-1} g_{k}}
$$

where again, $\Delta v$ is not of direct interest. In eqn.(17), only a single additional forward and backward substitution is required. Thus the computational effort is comparable to that of the inverse power method.

It should be pointed out that, the inverse power method and Newton's method are theoretically equivalent (see
Ref.[10]). But, the numerical properties they possess are quite different. For Newlon's method, convergence is guaranteed to be quadratic if it i:: started in the neighbourhood of the solution and the problem is almost always well-conditioned. Therefore, it is reasonable to use it following the inverse power method.

\section{Deflation}

In a large power system many eigenvalues can be very close to each other, as we will demonstrate in our test example. Therefore, sometimes it is difficult to differentiate between them and an eigenpair may be found repeatedly, while a nearby one would not be found at all. This difficulty is normally overcome by deflating procedures. The approach used in STEPS is based on a method described in Ref.[8], pages 595599. It is used in conjunction with the inverse power method, as follows.

First we solve eqn.(9) for $x_{k+1}$. Then we remove from $x_{k+1}$ its component in the direction of the known eigenvector, say $x_{1}$, using the equation

$$
x_{k+1}^{n e w}=x_{k+1}-\theta x_{1}
$$

where $\theta$ denotes the element of $x_{k+1}$ in the position where $x_{1}$ has its maximum element (normalized to unity). This ensures that the process will not converge to $x_{1}$.

The above process can be applied sequentially for several known eigenvectors, as shown in detail in Ref.[8].

\section{Participation Factors}

In order to assess the dominance of an eigenvalue, we evaluate the relaled participation factors. For this the left eigenvector has to be calculated.

Once an eigenvalue $\lambda$ and the associated right eigenvector $x$ lave been found, we can easily obtain the left eigenvector $y$ by the following procedure. By definition, $y$ satisfies the equation

$$
\left[\begin{array}{ll}
y^{T} & v^{T}
\end{array}\right]\left[\begin{array}{cc}
J_{A}-\lambda I & J_{B} \\
J_{C} & J_{D}
\end{array}\right]=\left[\begin{array}{ll}
0 & 0
\end{array}\right]
$$

with the constraint $y^{T} x=1$. Since the matrix in eqn.(19) is singular, we combine this equation with the constraint as follows:

$$
\left[\begin{array}{ll}
y^{T} & v^{T}
\end{array}\right]\left[J+x^{*} e^{T}\right]=e^{T}
$$

where $x^{*}$ is obtained by augmenting the right eigenvector $x$ and modifying it so that $J$ has the same form as $J_{k}$ in eqn.(16). Applying the Matrix Modification Lemma again for solving eqn.(20) yields

$$
\left[y^{T}-v^{T}\right]=c^{T} J^{-1}-\frac{\left(e^{T} J^{-1}\right)\left|x^{* T}\left(e^{T} J^{-1}\right)^{T}\right|}{1+x^{* T}\left(e^{T} J^{-1}\right)^{T}}
$$

Thus, the only significant computation needed is for evaluating $e^{T} J^{-1}$, which is done by an algorithm analogous to the one in Appendix 2. It is even possible to make use of the factorized submatrices of $J_{k}$ from the last iteration in Newton's method since, for the purpose of calculation of the participation factors, $y$ does not have to be too accurate. Therefore, only the forward and backward substitutions have to be performed, so that the procedure is very fast.

The left and right eigenvectors are used for the calculation of the participation factors as shown in Appendix 1. 


\section{PROBLEMS OF PRACTICAL IMPLEMENTATION}

\section{Description of Test System}

In order to verify STEPS, we have chosen a test system big enough to make an external area meaningful, in addition to a buffer around the study zone, as shown in Fig. 1. Its size had to be, however, limited such that a complete eigenanalysis, based on the QR algorithm, could be performed for the purpose of comparison.

The system used has 117 buses and 30 machines. It has been formed by combining three 39 bus systems derived from the New England system. The machine model used is of order 9 (a higher order model could have been chosen), with 6 state variables for the machine proper, 1 for the voltage regulator, and 2 for the stabilizer. Thus the complete dynamic order is $30 \times 9=270$. The sparse augmented system matrix, as in eqn.(5), is then $504 \times 504$

One of the 39 bus component systems has been chosen as nucleus, with two machines in the study zone. Thus the problem sizes in Stage 1 are:

10 - for the calculation of the mechanical modes,

26 - for the calculation of the slow modes,

26 - for the calculation of the fast modes.

We see then that the $\mathrm{QR}$ algorithm has to be performed for a matrix of at most $26 \times 26$, while for a full scale study the size of the matrix is $270 \times 270$. Since with the increase of the system size, the size of the nucleus could remain almost the same, the efficiency of STEPS would be even more pronounced. Of course, for really large systems the full size analysis by the $\mathrm{QR}$ algorithm is impractical.

\section{Selection of First Guess for Stage 1}

The selection of a set of first guesses to Stage 1 is quite arbitrary, provided that the values are well separated. Their number should be sufficient to assure that all desired eigenpairs will be found, but not excessive to keep the volume of computations as low as possible.

We have found appropriate the following first guess of 8 eigenvalues for our test system.

-For mechanical modes: $0+j 2 \pi$.

-For slow modes: $0+\mathrm{j} 0,0+\mathrm{j} 0.1,0+\mathrm{j} 0.5,0+\mathrm{j} 1.0$.

-For fast modes: $-1+\mathrm{j} 1,-2.5+\mathrm{j} 0,-5+\mathrm{j} 0$.

These values have been chosen to adequately cover the shaded regions of Fig. 2 where the eigenvalues are located. Their number should have been increased if the dominant modes would not all have been found.

\section{Selection of Starting Values for Stage 2}

Each first guess used as input to Stage 1 produces a full range of starting values by the process of Stage 1 . Clearly, only a subset of these should be chosen as inputs to Stage 2 . In STEPS we use the following selection criteria.

- For mechanical modes we use all eigenpairs obtained from Stage 1 , with the mechanical mode first guess $0+j 2 \pi$.

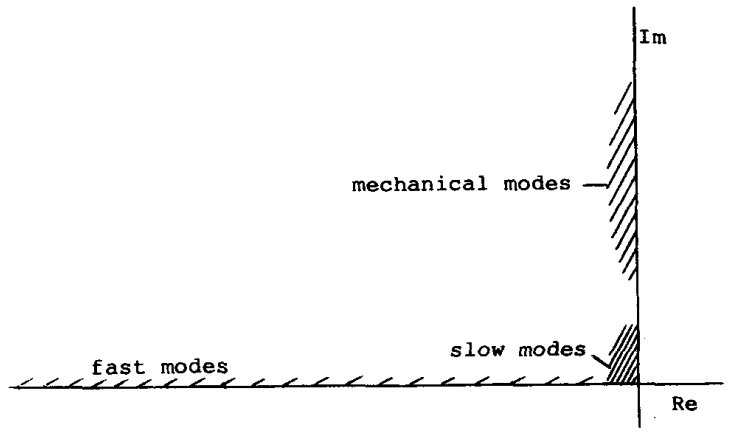

Fig. 2 Distribution of eigenvalues (conjugates not shown)

- For slow modes we use those eigenpairs, obtained from Stage 1 with the slow mode inputs shown in the previous section, which satisfy the following conditions:

$$
-5.0<\operatorname{Re}(\lambda)<0.2
$$

and

$$
|\operatorname{Im}(\lambda)|<5.0
$$

- For fast modes we use those eigenpairs, obtained from Stage 1 with the fast mode inputs shown in the previous section, which satisfy the following conditions:

$$
-\infty<\operatorname{Re}(\lambda)<-0.5
$$

and

$$
|\operatorname{Im}(\lambda)|<5.0 \text { or }|\operatorname{Im}(\lambda)|>15.0
$$

We note that the dimension of the eigenvectors in Stage 2 is bigger than in Stage 1 . Since the initial vector for the inverse power method could be almost arbitrary without seriously affecting convergence, we have formed it by augmenting with zeros the eigenvector obtained from Stage 1. This appears to be a reasonable choice.

\section{Strategy Used in Stage 2}

For fast and reliable convergence to the desired eigenpairs in Stage 2, the following approach has been adopted in STEPS.

a) If a starting value from Stage 1 is close to several already known eigenvalues (up to 3 ), then and only then, we deflate for those eigenpairs to ensure convergence to a different eigenpair.

b) We use the inverse power method to obtain an estimate for the eigenpair sufficiently close to the true value. It is important not to change the original shift in the first few iterations, in order to avoid drifting away from the desired target, as explained previously.

c) We apply Newton's method to hone in on the exact solution.

d) As a final step, we assess the significance of the eigenpair obtained, possibly by checking the elements in the eigenvector, related to the state variables of the study zone, or preferably, by calculating the participation factors related to these state variables. In this latter case we have the advantage that we can monitor whether all dominant modes have been identified. This is achieved by adding all participation factors related to a state variable as each 
mode has been calculated. Since, according to eqn.(A-5), the sum of all participation factors for a state variable is unity, the computation can be considered complete as soon as all running sums are sufficiently close to unity. We have considered an eigenvalue as dominant if at least one of the calculated participation factors has exceeded the threshold value $\alpha=0.05$. Therefore, theoretically, the running sums should get close to 0.95 to assure that all dominant modes have been found. This however is in practice not necessary and the calculations can be stopped earlier, with significant computational savings. We have found that when the running sums have reached 0.90 (i.e. $1-2 \alpha$ ) all dominant modes have been obtained.

\section{Results}

For the test system and the set of first guesses indicated above, we have obtained using STEPS a total of 73 different eigenpairs (some of them are complex), of which 39 are dominant in the 18 stale variables of the study zone. Since for complex eigenpairs, we only need to know any one of the two coirjugates, we have actually obtained 43 dominant eigenpairs containing 33 modes. We have checked, by comparing the set of dominant eigenvalues with the list of all 270 eigenvalues obtained from the full-size eigenanalysis of the system, that these constitute the complete eigensystem of the study zone, as defined by the $5 \%$, participation factor criterion. To save space, we list only some of them, namely the mechanical eigenvalues and a few eigenvalues which are very close to each other.

Table 1 contains the starting and converged values for the dominant mechanical eigenvalues, with the first guess $0+j 2 \pi$. It also gives the number of iteration steps for the inverse power method and for the subsequent Newton's method, with an error tolerance of $10^{-6}$. We note that the inverse power method required between 4 to 7 iterations, while only 2 or 3 steps were needed in Newton's method. An additional step would reduce the error to almost machine precision. From Table 1, it is clear that STEPS is very efficient for finding the mechanical eigenvalues related to the study zone, as this is usually the main purpose of the small signal stability analysis.

Table 1 Dominant Mechanical Eigenvalues in Study Zone

\begin{tabular}{|c|c|c|c|}
\hline Starting Value & Converged Value & \multicolumn{2}{|c|}{$\begin{array}{c}\text { Number of } \\
\text { Iterations }\end{array}$} \\
\hline$-0.455694+\mathrm{j} 9.367402$ & $-0.488755+\mathrm{j} 9.588277$ & 7 & 3 \\
$\mathbf{- 0 . 3 4 2 5 0 2}+\mathrm{j} 7.213383$ & $-\mathbf{0 . 4 6 4 8 0 0}+\mathrm{j} 7.464325$ & 6 & 3 \\
$-0.281357+\mathrm{j} 6.612058$ & $-\mathbf{0 . 3 3 7 6 4 2}+\mathrm{j} 6.736173$ & 4 & 2 \\
\hline
\end{tabular}

* The first column contains the number of iterations in inverse power method (including deflation, if any), and the second in Newton's method.

Table 2 shows two groups of the dominant eigenvalues which are very close to each other (error tolerance $=10^{-6}$ ). Since in this case deflation is always necessary, the number of iterations in the inverse power method was on the high side (5 to 11). As these are first performed without modifying the shift, no refactorizalion is needed, which makes the solution at that stage faster.
Table 2. Some Very Close Dominant Eigenvalues in Study Zone

\begin{tabular}{|r|c|c|c|c|}
\hline First Guess & Starting Value & Converged Value & \multicolumn{2}{|c|}{$\begin{array}{c}\text { Number of } \\
\text { Iterations* }\end{array}$} \\
\hline $0.0+\mathrm{j} 1.0$ & $-0.702607-\mathrm{j} 0.001072$ & $-0.703483+\mathrm{j} 0.000000$ & 5 & 2 \\
$0.0+\mathrm{j} 0.5$ & $-0.702267-\mathrm{j} 0.012730$ & $-0.703461-\mathrm{j} 0.000007$ & 6 & 7 \\
\hline $0.0+\mathrm{j} 1.0$ & $-0.732167+\mathrm{j} 0.007400$ & $-0.735510+\mathrm{j} 0.000000$ & 9 & 6 \\
$-2.5+\mathrm{j} 0.0$ & $-0.739836+\mathrm{j} 0.000000$ & $-0.735522+\mathrm{j} 0.000000$ & 7 & 4 \\
$-1.0+\mathrm{j} 1.0$ & $-0.740773-\mathrm{j} 0.002583$ & $-0.736452+\mathrm{j} 0.000000$ & 11 & 3 \\
\hline
\end{tabular}

* See footnote of Table 1 .

Table 3 lists all low frequency, interarea modes which are part of the dominant modes in the study zone. It took up to 14 inverse iterations and only 1 or 2 Newton iterations to compute these modes with an error tolerance of $10^{-6}$. We note that they have been calculated with the external system modelled with the same detail as the nucleus. This is of significance for the accuracy of the results.

Table 3. Interarea Oscillatory Modes in Study Zone

\begin{tabular}{|c|c|c|c|c|}
\hline First Guess & Starting Value & Converged Value & \multicolumn{2}{|c|}{$\begin{array}{c}\text { Number of } \\
\text { Iterations }\end{array}$} \\
\hline $0.0+\mathrm{j} 0.5$ & $-0.624340+\mathrm{j} 1.395198$ & $-0.446409+\mathrm{j} 1.344450$ & 14 & 1 \\
$0.0+\mathrm{j} 0.5$ & $-1.058608-\mathrm{j} 1.319131$ & $-0.686389-\mathrm{j} 1.235600$ & 11 & 2 \\
$0.0+\mathrm{j} 1.0$ & $-0.451743-\mathrm{j} 1.352466$ & $-0.455627-\mathrm{j} 1.356922$ & 6 & 1 \\
$0.0+\mathrm{j} 1.0$ & $-0.577766+\mathrm{j} 1.341575$ & $-0.658292+\mathrm{j} 1.231110$ & 12 & 1 \\
$0.0+\mathrm{j} 2 \pi$ & $-0.121715+\mathrm{j} 1.707151$ & $-0.406807+\mathrm{j} 1.641528$ & 12 & 1 \\
\hline
\end{tabular}

* See footnote of Table 1 .

For all 39 dominant eigenvalues the number of iterations for the inverse power method has never exceeded 15 (mostly without updating the shift), and for Newton's method it was never more than 9 (usually below 4). Obviously, the former has linear convergence, and the latter quadratic.

The computing time for the test example was 32.4 minutes on an IBM 4361 running CMS (in double precision). If one is interested in a particular set of eigenvalues, the computing time required can be estimated as being approximately proportional to the number of first guesses needed for Stage 1 . In the case of mechanical eigenvalues, this would be $1 / 8$, i.e. $\approx 4$ minutes (the exact time was 3.2 minutes). As a comparison, a full-scale eigenanalysis of the test system by the $Q R$ algorithm (we have used EISPACK routines) requires 47 minutes.

More important than the reduced computing time is the modest storage requirement, due to the sparse implementation of all relevant parts of the procedure. For the test system, the storage requirement of STEPS is approximately $1 / 5$ of that of the full-scale analysis, and it will not increase much if the system becomes larger. Therefore, STEPS could be considered most suitably for the small signal stability analysis of large power systems.

\section{CONCLUSIONS}

The paper has presented a successful implementation of procedures which permit the sequential computation of all eigenpairs which are dominant in the state variables of the study zone of a large power system. The program, called STEPS (Sequential Two-stage Eigenanalysis of Power Systems), obtains first an approximation to the eigenpairs, then refines them to the desired precision. The following are the main features of STEPS: 
a) All dominant eigenvalues of the study zone can be found, even if they are very close to each other.

b) The storage requirements for even large systems are reduced, due to sparse matrix procedures.

c) If only particular eigenvalues are sought, the computing time is reasonably small. Especially, if only mechanical eigenvalues of the study zone are of interest, they can be found easily with a few iterations.

d) The representation of the external system in full detail permits the accurate calculation of the interarea oscillatory modes which may be sensitive to the modelling of all system components.

e) Since in Newton's method employed in STEPS, the Jacobian can be considered a system sensitivity matrix, it can be used for analyzing the effect of control parameters on small signal stability.

\section{ACKNOWLEDGEMENTS}

The authors wish to acknowledge financial support by the Natural Sciences and Engineering Research Council of Canada. The second author also wishes to express his thanks to Shanghai Jiao Tong University, People's Republic of China, for supporting his studies at the University of Toronto.

\section{REFERENCES}

[1] P. Kundur and P.L. Dandeno, "Practical Application of Eigenvalue Techniques in the Analysis of Power System Dynamic Stability Problems", Proc. Fifth Power Computation Conference, Cambridge, Englarid, September 1975.

[2] Y. Obata, S. Takeda, and H. Suzuki, "An Efficient Eigenvalue Estimalion Technique for Multi-Machine Power System Dynamic Stability Analysis", IEEE Trans. Vol. PAS-100, pp 259-263, January 1981.

[3] R.T. Byerly, R.J. Benon, and D.E. Sherman, "Eigenvalue Analysis of Synchronizing Power Flow Oscillations in Large Electric Power Systems", IEEE Trans. Vol. PAS101, pp 235-243, January 1982.

[4] P. Kundur, "Evaluation of Method for Studying Power System Stability", International Symposium on Power System Stability, Ames, Iowa, U.S.A, May 1985.

[5] G.C. Verghese, 1.J. Perez-Arriaga, and F.C. Schweppe, "Selective Modal Analysis with Application to Electric Power Systems, Part II: The Dynamic Stability Problem", IEEE Trans. Vol. PAS-101, pp 3126-3134, September 1982.

[6] N. Martins, "Efficient Eigenvalue and Frequency Response Methods Applied to Power System Small-Signal Stability Studies", IEEE Trans. Vol. PWRS-1, pp 217-226, February 1886.

[7] D.Y. Wong, G.J. Rogers, B. Porretta, and P. Kundur, "Eigenvalue Analysis of Very Large Power Systems", IEEE Paper No. 87 WM 102-7.

[8] J.H. Wilkinson, The Algebraic Eigenvalue Problem, Clarendon Press, Oxford, 1965.

[9] D.K. Faddecr and V.N. Faddeeva, Computational Methods of Linear Algebra, W.H. Freeman and Company, 1963.
[10] G. Peters and J.H. Wilkinson, "Inverse Iteration, IIIconditioned Equations and Newton's Method", SIAM Review, Vol. 21, pp 339-360, July 1979.

[11] H.V. Henderson and S.R. Searle, "On Deriving the Inverse of a Sum of Matrices", SIAM Review, Vol. 23, pp 53-60, January 1981.

\section{APPENDICES}

\section{Appendix 1 - Participation factors}

Let

$$
x(t)=e^{A t} x(0)
$$

be the zero input solution of a dynamic system with the state vector $x$, or

$$
x(t)=\Phi e^{\Lambda t} \Psi x(0)
$$

where

$\Phi$ is the matrix of the right column eigenvectors of $A$,

$\Psi=\Phi^{-1}$ is the matrix of the normalized left row eigenvectors of $A$,

$\Lambda$ is the diagonal matrix of the eigenvalues of $A$.

We focus on the single state variable $x_{k}$, by setting $x_{k}(0)=1$ and $x_{j \neq k}(0)=0$. Then we obtain

$$
x_{k}(t)=\sum_{i} \phi_{k i} \psi_{i k} e^{\lambda_{i} t}
$$

This equation shows that mode no.i, as excited by the initial value $x_{k}(0)=1$, participates in the response $x_{k}(t)$ with a coefficient

$$
p_{k i}=\phi_{k i} \psi_{i k}
$$

called participation factor. In eqn.(A-4), $\phi_{k i}$ and $\psi_{i k}$ represent the element no.k of the ith right and left eigenvector of matrix $A$, respectively.

We note that, for $t=0$, eqn.(A-3) gives

$$
\sum_{i} p_{k i}=1
$$

\section{Appendix 2 - Solution of the Sparse Augmented Linear Algebraic Equations}

In the method presented in this paper, we need to solve the sparse augmented linear algebraic equations of the form

$$
\left[\begin{array}{cc}
J_{A}-\lambda I & J_{B} \\
J_{C} & J_{D}
\end{array}\right]\left[\begin{array}{l}
x \\
v
\end{array}\right]=\left[\begin{array}{l}
w \\
u
\end{array}\right]
$$

as eqn.(8) and (10). We use the following algorithm in STEPS to obtain the solution $x$ and $v$.

a) Calculate

$$
J_{D_{* 1}}=J_{D}-J_{C}\left(J_{A}-\lambda\right)^{-1} J_{B}
$$

We note that $\left(J_{A}-\lambda I\right)$ is block-diagonal and $J_{D_{a,}}$ has the same sparse structure as $J_{D}$.

b) Solve for $v$

$$
J_{D_{\text {q }} v} v=u-J_{C}\left(J_{A}-\lambda\right)^{-1} w
$$

c) Solve for $x$

$$
\left(J_{A}-\lambda I\right) x=w-J_{B} v
$$




\section{Discussion}

Ovidiu Crisan (University of Houston, Houston, TX): I would like to congratulate the authors for their excellent paper. Their procedure is ingenious and practical with positive effects on computing time and storage requirements. It should also be mentioned that the capability of handling detailed component models increases the discerning power regarding different modes.

It would be appreciated if the authors could comment on the following questions.

I) It is mentioned in the paper that the size of the nucleus could remain almost the same for any size of the system. If the ratio of the sizes of the nucleus and of the system is too small, would the computing time for Stage 2 not be negatively affected? Could the authors give an estimation of the minimum acceptable value of this ratio?

2) The selection of the first guesses for Stage 1 was mentioned to be quite arbitrary. What is the effect on the computation time of the deviation of the first guesses with respect to their final values? Should one take care to select the first guesses within some acceptable range of their final values?

3) Depending on the power system load, in some components of the generator control channels of some units the variables may reach an external or internal limit. What effect will this have on the eigenvalues? Can the new values be determined in a faster way, knowing their values when all control channels are in a continuous operation domain?

J. H. Chow (Rensselaer Polytechnic Institute, Troy, NY): The authors are to be commended for their paper which presents a novel approach in the important field of eigenfunction analysis of large power systems. One of the contributions of the paper is a method of computing the initial guess of the eigenvalues (stage 1). A good initial guess is important since it reduces the stage 2 computation. To probe further, I pose the following questions.

1) It is desirable to provide an error estimate of an eigenvalue $\lambda$ computed in stage 1 . For example, is it possible to place a small disk centered at $\lambda$ and say that a true eigenvalue of the system is inside the disk?

2) What is the computation requirement of stage 1 compared to stage 2 ?

3) Some elaboration on how the problem sizes are selected in the test system will be helpful.

4) In case it is decided that some dominant eigenvalues are missing, what should one do next? Expand the nucleus? Repeat stage 1 with some of the true eigenvalues computed in stage 2 ?

The authors' comments to these questions will be appreciated.

Jia Yu Huang (Shanghai Jiao Tong University, Shanghai, The People's Republic of China): The important contribution of this paper is having developed a new method and program for calculating the complete eigensystem of the study zone in the small signal stability study of a large power system. In the new method the whole system is divided into two parts, the computation is performed in two stages and Newton's method as well as deflation are used together with the inverse power method. The techniques used make it possible to study the behaviors in a certain region of a large power system thoroughly, to obtain the complete eigensystem of the study zone accurately and reliably, and to save the computer time and storage substantially. These techniques constitute the characteristics of the new method.

I would like to remark that the starting values in Stage 2 are close to the converged values though they are far from the first guesses in Stage 1. The results in the three tables are interesting and valuable.

I feel that the reasoning why the whole system is not necessarily represented accurately in Stage 1 is not very clear. I would also appreciate it if the result of a complete eigensystem could be listed and an example of applying the new method to improve the dynamic stability of an unstable power system could be offered further.

In closing, I would like to congratulate the authors for their fine paper.

Nelson Martins (CEPEL, Rio de Janeiro, Brazil): The authors are to be congratulated on this valuable paper. They have used and further developed many ideas conveyed in recent literature [2], [5], [6]. With regard to the concepts and algorithms used in the computer program STEPS, the discusser would like to make the following comments and questions.

1) The concepts of study zone, buffer area, and external system are very valuable to static network analysis. However, in small signal stability analysis, this concept may only be applied after careful study done by specialists on dynamic equivalencing. The reason is that interarea oscillatory modes generally spread over the entire interconnected system. Also the proposed eigensolution of a specified study zone may not reveal unstable or undamped eigenvalues which happen to be mainly related to other areas of the system (i.e., with participation factors less than 0.05 for states of the study zone).

Could the authors please explain the main applications they envisage for STEPS?

2) The discusser has used inverse iteration to solve large practical systems (about a thousand states) and has not detected wrong answers due to ill-conditioned equations. It may however be comforting to check for a small residue $r_{k}=\left(A-\lambda^{k} I\right) x_{k}$ at convergence (where $k$ is the iteration number).

When using Rayleigh quotient correction for the eigenvalue estimate at every iteration the discusser assumes convergence when a small diagonal pivot $\left(10^{-6}\right.$ or $\left.10^{-7}\right)$ occurs during factorization of $\left(A-\lambda^{k} I\right)$. It may be added that the discusser's programs work on double precision arithmetic.

Reference [10] emphasizes that if $\lambda^{k}$ is corrected each time, the inverse iteration algorithm is every bit as satisfactory as Newton's method. Inverse iteration can take full advantage of the special form of the augmented system state equations, while the authors have had to resort to a more elaborate formulation to preserve sparsity in Newton's method.

3) In order to assure diagonal dominance of matrix $J_{D}[6]$, the network equations must be arranged such that the vector of injected currents in (4b) is ordered $\left(I_{i 1}, I_{r i} \cdots I_{i n}, I_{m}\right)^{t}$

4) When solving equation (A-6) for very large problems, one notes that the calculation of complex matrix $J_{\text {Deq }}$ (step " $a$ " of Appendix 2) is fast, while the solution for $v$, which involves the factorization of $J_{\text {Deq }}$ (step " $b$ "), is very time consuming. Expressing the network equations in $P, N$ variables rather than $D, Q$ variables may partly alleviate this problem [7]. In order to further decrease computation time, the network should be reduced but only as long as the equivalent network remains very sparse.

5) This item presents another alternative scheme for the inverse iteration problem.

Apply inverse iteration to the well-conditioned Hermitian matrix $C=(A$ - $\left.\lambda^{k} I\right)^{t}\left(A-\lambda^{k} I\right)$, which necessarily has an orthogonal set of eigenvectors. In the present application, the eigenvalue estimates are only approximately known, and therefore many iterations are normally needed for convergence. Inverse iteration applied to matrix $C$ requires additional computation per iteration, but the extra work may be offset by the stronger convergence behavior of the problem since $\lambda_{C}=\left(\lambda_{A}-\lambda_{k}\right)^{2}$. For clarity, some definitions are necessary here: $\lambda_{k}$ is the eigenvalue estimate, while $\lambda_{A}$ and $\lambda_{C}$ are the exact eigenvalues of matrices $A$ and $C$, respectively.

6) Equations (19) to (21) describe a procedure for finding the lef eigenvector. A simpler procedure, using the same $L U$ factors of $J_{A}$ and $J_{\text {Doq }}$, can be derived by noting that $A^{t}=U^{t} \cdot L^{t}$.

7) Eigenvalue sensitivity is given by the well-known formula:

$$
\frac{\partial \lambda_{i}}{\partial \alpha}=\frac{\left(\frac{\partial A}{\partial \alpha} x_{i}, v_{i}\right)}{\left(x_{i}, v_{i}\right)}
$$

where $\alpha$ is a system parameter and $x_{i}$ and $v_{i}$ are the right and left eigenvectors of $\lambda_{i}$. The state matrix derivative is given by:

$$
\frac{\partial A}{\partial \alpha}=\frac{\partial}{\partial \alpha}\left(J_{A}-J_{B} J_{D}^{-1} J_{C}\right)
$$

The discusser could not completely understand the remarks made on the analysis of the control parameter effects based on the matrix of Newton's method. Could the authors please explain?

M. Eremia and C. Aldea (Polytechnical Institute of Bucharest, Bucharest, Romania): We wish to congratulate the authors for developing some original procedures to find eigenpairs for large power systems. These procedures yleld very accurate numerical results and they keep memory and computational time within acceptable limits.

This methodology, taking into consideration results obtained by other research as well, makes use of some special techniques of modal analysis and linear algebra. Thus, it is able to overcome the limitations imposed by classical engineering in analyzing large power systems.

We remark on the possibility of using the computer program STEPS in stability analysis for small perturbations of different controlling parameters influence. This problem is of large interest for us, too. We would like to know if the sensitivity matrix which is defined by the Jacobian matrix $B$ can be used with the same results and efficiency as the matrix defined in [1]. 
References

[1] J. E. Van Ness, J. M. Boyle, and F. P. Imad, "Sensitivities of Large Multiple-Loop Control Systems," IEEE Trans. Automatic Control, vol. AC-10, July, 1965.

Fernando L. Alvarado (The University of Wisconsin, Madison, WI): This is a paper of potentially broader applicability than merely power systems. Three key ideas in the paper worthy of further scrutiny are the idea of explicit retention of algebraic equation network structure within the eigenvalue formulation, the idea of the use of approximate explicitly assumed eigenvalues for the portions of the system not directly of interest based on participation factors, and the idea of Newton refinement of eigenvalues.

The first idea, explicit retention of algebraic structure, is expected to help retain sparsity in much the same way as not reducing systems helps retain sparsity during ordinary network equivalencing and reduction. A number of references have described the impact of reduction on sparsity [A1]-[A3]. Do the authors have any experience on the sparsity implications of their method?

The second idea, the use of approximate explicit eigenvalues for portions of the system not of interest, is interesting. Do the authors have any experience on possible limitations of this concept? Can they cite any similar attempts in the mathematical or engineering literature?

The third idea, the use of Newton's method for refinement of eigenvalues, is not entirely new. However, this discusser would like to refer the authors to a recent paper [A4] that presents some ideas about general purpose symbolically assisted numeric computation that may make Newton refinement of solutions to any problem a quite trivial task from the implementation viewpoint. In fact, this discusser would welcome any thoughts the authors may have on the applicability of the program described in [A4] toward the iterative refinement of eigenvalues.

In short, this paper is quite interesting, and this discusser commends the authors for their effort.

\section{References}

[A1] S. Deckman, A. Pizzolante, A. Monticelli, B. Stott, and O. Alsac, "Studies of Power System Load Flow Equivalents,"' IEEE Trans. Power App. Syst., vol. PAS-99, No. 6, pp. 2301-2310.

[A2] W. F. Tinney and J. M. Bright, "Adaptive Reductions for Power Flow Equivalents," IEEE Winter Power Meeting, Paper 86 WM $097-0,1986$

[A3] F. L. Alvarado and E. H. Elkonyaly, "Reduction in Power Systems," IEEE PES Summer Meeting, Mexico City, July 1977, paper A77 507-7.

[A4] F. L. Alvarado and Y. Liu, "General Purpose Simulation Tools for Electric Networks," Proc. 1987 PICA Conf., Montreal, Canada, May 18-22, 1987, pp. 222-229.

Adam Semlyen and Lei Wang : We would like to thank the discussers for their interest in our paper and for their questions and comments. Many of these constitute contributions to the topic of the paper. We will give our answers to each discusser separately.

Dr. O. Crisan:

1) The size of the nucleus depends mainly on the eigenvectors associated with the dominant modes in the study zone. It can be shown ${ }^{A}$ that the procedure in Stage 1 is convergent if

$$
\frac{\left\|x_{2}\right\|}{\left\|x_{1}\right\|}<1
$$

where $x=\left[\begin{array}{ll}x_{1}^{T} & x_{2}^{T}\end{array}\right]^{T}$ is the right-hand eigenvector of a dominant mode in the study zone and $x_{1}$ is its portion corresponding to the state variables in the nucleus. In eqn.(a), the norm is taken with respect to the left-hand eigenvector of the same mode; see Ref.[A]. This result implies that for the calculation of dominant modes in the study zone, a small buffer area is adequate, since in this case $x_{1}$ is dominant no matter how big the whole system is. A minimum acceptable nucleus could be determined by eqn.(a).

2) We agree with the discusser that the first guesses should be within a reasonable range of their final values, for example, distributed uniformly in three typical regions representing mechanical, slow, and fast modes. We have found that these very rough guesses could, rather surprisingly, produce quite good estimates for the eigenvalues dominant in the study zone. In most cases, the error between the starting values (from Stage 1) and the true values is within $5 \%$. The computation time depends very little on the accuracy of the first guess.

3) We note that, in practice, there are two kinds of limits for generator control variables: operational and physical. The former have simply no effect on the dynamic performance of the system, since it is always possible for any variable to have a small increment above its operational limit. In the latter case, however, the variable becomes a constant, so that it will not appear in the state equation. This will certainly change the eigenvalues of the system. Even though we have not explored this topic, we have the feeling that the ncw eigenvalues might be very close to the old ones and, therefore, easy to be calculated.

Dr.J.H. Chow:

1) As mentioned above, the results from the test system show that the starting values obtained from Stage 1 are in most cases within $5 \%$ with respect to the true eigenvalues. This makes us believe that, after finding an eigenvalue $\lambda$ in Stage 1 , there is likely a true eigenvalue in a disc, centered at $\lambda$ with the radius of $5 \%$ of $|\lambda|$.

2) The computational requirement of Stage 1 is much less than that of Stage 2. For the test system, the CPU time for Stage 1 is about 5 minutes, or $15 \%$ of the total CPU time. This is because the main computation in Stage 1 consists of only a few $Q R$ procedures applied to the reduced problem which is small, while in Stage 2 we have to handle the complete set of system equations which has very big size even though it is very sparse.

3) The way of forming the reduced problems in Stage 1 is explained in the paper. For the test system, three reduced problems are generated as follows:

(a) For mechanical modes, neglect all machine frictional losses, keep only the mechanical equation for each machine in the nucleus, and eliminate all other equations using the first guess $\lambda_{0}$. This gives 10 final state equations in the standard form.

(b) For slow modes, keep all 18 state variables in the study zone and 8 additional machine angles in the nucleus, and eliminate all other equations in the same way as for mechanical modes. So the problem size is 26 .

(c) For fast modes, everything is the same as for slow modes, except that $\delta$ is replaced by $\omega$. Therefore the problem size remains unchanged, i.e. 26.

4) In case that some dominant eigenvalues are missing, one could simply add one or more first guesses which should be different from the rest, until all dominant eigenvalues are found.

Prof. J. Y. Huang:

We review in some detail the basic idea of the paper in order to clarify the algorithm we have developed.

From the point of view of small signal performance, power systems can be considered as weakly coupled dynamic subsystems since the system-wide coupling between state variables is mainly through $\delta$ and $\omega$ via transmission links. This fact can also be observed by noting that in the state matrix of power systems, most of the elements are extremely small, even though the matrix itself is non-sparse (for example, in the state matrix of our test system, $93.65 \%$ of all elements are less than $0.1 \%$ of the biggest value in the matrix). Also, for machines far from the study zone, the coupling with the study zone is small by not being direct, so that it is reasonable to use rough first guess eigenvalues all over in that region. For the machines surrounding the study zone (i.e. in the buffer area), it is possible to make other approximations. Firstly, we can use approximations for the eigenvalues of all non-mechanical state variables. Secondly, depending on the type of modes to be determined, we have different strategies. If mechanical modes are of interest, we keep only the mechanical equations which become strongly dominant in this case. This leads to a reduced problem of onder equal to the number of machines in the nucleus (for each machine, the angular velocity $\omega$ can be eliminated after neglecting frictional losses). For slow and fast modes, we see from

$$
\frac{d \delta}{d t}=\omega
$$

or, in the eigenvalue problem,

$\lambda \delta=\omega$

that, if $\lambda$ is small then $\delta>\omega$ (i.e. $\delta$ is more important than $\omega$ for slow 
modes), and if $\lambda$ is big then $\omega>\delta$ (i.c. $\omega$ is more important than $\delta$ ). Since, as shown in Ref.[A], only the dominant state variables need to be kept in the reduced problem, we leave only $\delta$ 's or $\omega$ 's for slow or fast modes, respectively, outside the study zone, while retaining all state variables inside it.

We regret that, due to limited space, we are not able to list here the detailed results we.haye obtained for the test system. However, we would be pleased to communicate privately with any interested reader. We do not yet have results conceming the stabilization of an unstable system.

\section{Dr. N. Martins:}

1) In our view, the study zone is more a geographical than analytical concept, so that in STEPS one does not have to perform a preliminary analysis to produce a study zone; instead, one may simply circle in the area of interest. The purpose of this paper is then to find all the dominant eigenvalues for that area. We agree with the discusser that inter-area oscillatory modes usually spread over the entire system and, quite possibly, some unstable or lightly damped eigenvalues, dominant in some other portion of the system, will not actively participate in the study zone. For the former, results of STEPS have shown that, whenever a mode (inter-area or local) participates in the study zone strongly, it can be found, while weakly participating modes (no matter whether interarea or local) are, in our opinion, of little interest for the study zone, unless the problem of the stability of the whole system is examined.

Regarding the applications of STEPS, we envisage the following:

(a) STEPS could be used, in conjunction with other programs such as parameter sensitivity analysis, to design and tune the control systems of specified machines (and possibly HVDC links). In this case, modes which do not participate actively in the interested area are of no significance.

(b) In some early papers (for instance [B]), a method with an electromechanical equivalent was proposed for the calculation of transient stability. The method has not been used widely because it needs the detailed modal analysis for the area to be equivalenced, which is difficult for large power systems. With STEPS, however, this can be done efficiently, which makes the method more attractive.

2) The comparative analysis of the characteristics of inverse iteration and Newton's method is a most interesting problem. We agree with the discusser that to a large extent they are equivalent, but they do have some differences with regard to convergence, as illustrated below.

Consider the matrix

$$
A=\left[\begin{array}{cc}
1.0 & \alpha \\
\beta & 1.0
\end{array}\right]
$$

where we assume $\alpha \beta=10^{-10}$. This matrix has the exact eigenvalues $0.99999,1.00001$ and its eigenvectors vary with the parameters $\alpha$ and $\beta$. Now, if we start at $\lambda_{0}=0.9$ and $x_{0}=[1.0-1.0]^{T}$ for:

(a) Inverse iteration,

(b) Inverse iteration for the first three steps followed by Newton's method,

we obtain the following results for $\alpha=4 \times 10^{-3}$ and $\beta=0.25 \times 10^{-7}$ (single precision was used)

\begin{tabular}{|c|c|c|c|c|}
\hline Iter. No. & & Case: & & ase b \\
\hline$n$ & $\lambda_{n}$ & $\left\|A x_{n}-\lambda_{n} x_{n}\right\|_{2}$ & $\lambda_{n}$ & $\left\|A x_{n}-\lambda x_{n}\right\|_{2}$ \\
\hline 0 & 0.8999999760 & 0.1386217470 & 0.8999999760 & 0.1386217470 \\
\hline 1 & 0.9980015160 & 0.0026657861 & 0.9980015160 & 0.0026657861 \\
\hline 2 & 0.9988131520 & 0.0004107477 & 0.9988131520 & 0.0004107477 \\
\hline 3 & 0.9993910790 & 0.0000961238 & 0.9993910790 & 0.0000961238 \\
\hline 4 & 0.9996937510 & 0.0000236583 & 0.9996917250 & 0.0000237307 \\
\hline 5 & 0.9998466370 & 0.0000058862 & 0.9998456840 & 0.0000059275 \\
\hline 6 & 0.9999227520 & 0.0000014705 & 0.9999225140 & 0.00000014759 \\
\hline 7 & 0.9999608990 & 0.0000003838 & 0.9999606010 & 0.0000003632 \\
\hline 8 & 0.9999790190 & 0.0000001466 & 90190 & 0.00000000864 \\
\hline 10 & 0.9999905820 & 0,000008161 & 0.9999896290 & 0.0000000507 \\
\hline ii & 0.9999905230 & 0.0000005159 & 0.9999899860 & 0.0000000078 \\
\hline 12 & 05230 & 0.0000005230 & 360 & 0.0000000136 \\
\hline 13 & 205230 & 0.0000005229 & 0.9999899860 & 0.0000000136 \\
\hline 14 & 005230 & 0.0000005229 & 0.9999899860 & 0.0000000136 \\
\hline
\end{tabular}

It can be seen from the table that upon final convergence, Newton's method can give up to 7 significant digits, while inverse iteration has jus 5 (note: there is no double precision calculation in Newton's method) Also, the final residue in Newton's method is much less than that in inverse iteration. These facts clearly indicate that Newton's method has higher accuracy than inverse iteration. Moreover, if we allow $\alpha$ and $\beta$ to vary continuously, we can observe that the convergence of Newton's method has almost the same pattern, while the convergence characteristics of inverse iteration are quite different: sometimes fast and accurate, sometimes slow and less accurate. This indicates that Newton's method is more stable and reliable. The reason for the above results was analyzed in Ref.[10]. It is due to the ill-condition of the eigenproblem of $A$ when $\beta$ is small. Unfortunately, the eigenproblem of power systems seems to be ill-conditioned, because of many close eigenvalues and possibly close-to-dependent eigenvectors.

On the other hand, if we check the volume of computation for both algorithms, we find that Newton's method is worth being considered. Except for the common calculation of solving linear equations, Newton's method needs one additional forward and backward substitution, as indicated in eqn.(17) by $J_{k}^{-1} g_{k}$, and the modified eigenvector is always normalized, while in inverse iteration, as suggested by the discusser, the calculation of the Rayleigh quotient requires first the normalization of the eigenvector $x_{k}$ then the computation of the quadratic form $x_{k}^{T} A x_{k}$ (which is nearly equivalent to calculating $J_{k}^{-1} g_{k}$ in Newton's method). Therefore, Newton's method is a bit simpler than inverse iteration, and it has the further advantage of permitting easy calculation of the left-hand eigenvector (eqns.(19) to (21)).

To summarize, we believe Newton's method is a good choice for refining eigenpairs. It compares favorably with inverse iteration, which itself has shown excellent characteristics as a first-stage refinement tool.

3) We fully agree with the discusser that the injected current vector should be re-ordered in the suggested form whenever this algorithm is to be used in practical applications. The arrangement in the paper is only for the simplicity of our test program.

4) We appreciate the comments of the discusser regarding the solution of the system equations.

5) In eqns.(19) to (21), the left-hand eigenvector is in fact found in the way the discusser suggested, except for a different representation for making use of the factorized matrix $J$ from the last Newton iteration. All the work to be done is forward and backward substitutions and one inner product calculation.

6) We would like to explain brieny what we mean by sensitivity analysis of the effect of control parameters based on matrix $B$ of Newton's method.

From the original eigenvalue problem

$$
A x=\lambda x
$$

we can obtain, by taking derivatives with respect to a control parameter

$$
\frac{\partial A}{\partial \alpha} x+A \frac{\partial x}{\partial \alpha}=\frac{\partial \lambda}{\partial \alpha} x+\lambda \frac{\partial x}{\partial \alpha}
$$

or

$$
(A-\lambda I) \frac{\partial x}{\partial \alpha}-\frac{\partial \lambda}{\partial \alpha} x=-\frac{\partial A}{\partial \alpha} x
$$

Using the same definition for matrix $B$ and for the vector $x^{\prime}$ as in eqn.(13), eqn.(e2) can be written as

$$
B \frac{\partial x^{\prime}}{\partial \alpha}=-\frac{\partial A}{\partial \alpha} x
$$

where $x$ is normalized in the same way as for Newton's method. From (f) we obtain

$$
\frac{\partial x^{\prime}}{\partial \alpha}=-B^{-1} \frac{\partial A}{\partial \alpha} x
$$

This is the required sensitivity equation based on matrix $B$ of Newton's method. We note that $(g)$ includes both the eigenvalue and eigenvector sensitivities while, with the classical formula (eqn.(i) below), only the eigenvalue sensitivity is obtained. In fact, it is easy to show that the eigenvalue sensitivity in (g) is exactly the same as from the classical formula. Without loss of generality, we assume that the first element of the right-hand eigenvector is normalized to unity, so that matrix $B$ has the form

$$
B=\left[-x(A-\lambda I)_{n, n-1}\right]
$$

where $(A-\lambda I)_{n, n-1}$ is the $n \times(n-1)$ matrix of the last $(n-1)$ columns of $(A-\lambda I)$. Further let 


$$
B^{-1}=\left[\begin{array}{c}
z^{T} \\
B_{n, n-1}^{T}
\end{array}\right]
$$

Then,

$$
B^{-1} B=\left[\begin{array}{cc}
-z^{T} x & z^{T}(A-\lambda I)_{n, n-1} \\
\times & \times
\end{array}\right] \equiv I
$$

where $X$ means a submatrix independent of vector $z$. We see from (h) that a vector $z^{T}$ can be the first row of $B^{-1}$ if and only if it satisfies

(a) $-z^{T} x \equiv 1$,

(b) ${ }_{2}^{T}(A-\lambda J)_{n, n-1} \equiv 0$.

Evidently, $z^{T}=-\frac{y^{T}}{y^{T} x}$ is the solution which satisfies both (a) and (b), where $y$ is the left-hand eigenvector of $A$. Substituting this solution into (g), we obtain

$$
\frac{\partial \lambda}{\partial \alpha}=\frac{y^{T} \frac{\partial A}{\partial \alpha} x}{y^{T} x}
$$

which is the classical eigenvalue sensitivity formula mentioned by the discusser.

Finally, if we make a comparison between (g) and (i), we can see that, since matrix $B$ is available directly from Newton's method in factorized form, the evaluation of $(\mathrm{g})$ is simpler than that of $(\mathrm{i})$ which requires the left-hand eigenvector.

Dr.M. Eremia and Dr. C. Aldea:

We thank the discussers for their valuable comments. As for the sensitivity analysis based on matrix $B$ of Newton's method, we would like to refer to our answer to Dr. Martins.

Dr.F.L. Alvarado:

1) The alternative to retaining the full set of system equations would have implied solving large sets of non-sparse equations. We believe that the first approach is superior but did not compare the efficiencies of the two methods.

2) The idea of using approximate eigenvalues related to the state variables in the extemal system appears already in Ref.[5] and its companion paper. As pointed out by the discusser, the particular structure of the power system, namely that it consists of subsystems coupled only through terminal variables, is a feature of many other large systems, biological, economical, engineering, and so on. Therefore, the eigenanalysis of the paper could be applied to such systems. We are not aware however of related literature from other fields of applications.

3) We wish to thank the discusser for bringing to our attention the general purpose simulation tools of Ref.[A4]. We would like to add Ref.[D] about an easy-to-use interactive general purpose computer program for solving (large) sets of nonlinear equations. Newton's method for solving the eigenvalue/vector problem of eqn.(11) is presented there as a simple, straightforward application.

\section{References}

[A] A. Semlyen and L. Wang, Discussion to Reference [7].

[B] J. M. Undrill, J. A.Casazza, E. M. Gulachenski and L. k. Kirchmayer, "Electromechanical Equivalents for Use in Power System Stability Studies", IEEE Trans. Vol. PAS-90, pp 2060-2071, Sept/Oct 1971.

[C] N. Uchida and T. Nagao, "A New Eigen-Analysis Method of Steady-State Stability Studies for Large Power Systems: S Matrix Method", presented at PICA 1987, Montreal, Canada.

[D] F. L. Alvarado, Solver-Q: Instruction Manual (version 1.01), University of Wisconsin, May 1987. 